\title{
A comparison of modified periodogram and Blackman-Tucky methods for wideband receiver applications
}

\author{
Jason Pennington ${ }^{1}$ and Chi-Hao Cheng ${ }^{2, \text { a) }}$
}

Abstract A wideband receiver is designed to detect signals over a broad bandwidth. To increase the receiver's capability of detecting multiple signals, it is a common practice to apply a window to reduce spectral leakage. The modified periodogram applies a window before auto-correlation and Blackman-Tucky method applies the window after auto-correlation. To the best of authors' knowledge, no one has compared the performance difference in the context of wideband receiver applications. This study finds that the modified periodogram can better enable a receiver to detect multiple signals with a significant power difference at the expense of reduced frequency resolution and sensitivity.

Keywords: wideband receiver, periodogram, Blackman-Tucky method

Classification: Microwave and millimeter-wave devices, circuits, and modules

\section{Introduction}

The wideband receiver like an electronic warfare receiver is designed to detect an unknown number of signals and determine their characteristics $[1,2,3,4,5,6,7,8,9]$. A simplified Fast Fourier Transform (FFT)-based wideband receiver is illustrated in Fig. 1. Here, the antenna continuously surveys the ambient environment. The received signal is then amplified, sampled and digitized by an analog-to-digital converter (ADC). Afterwards, pre-processing and FFT operation is applied to the sampled data and the signal frequency and power can be estimated from the FFT result. Signal characteristics such as signal frequency/chirp rate, signal power, time-of-arrival (TOA), pulse-width (PW), etc.-all of which are summarized in a Pulse Descriptor Word (PDW) [1]. Because of the wide working bandwidth, a wideband receiver might have multiple channels [7, 10] and Fig. 1 shows only one channel. Due to its computational efficiency, the FFTbased EW receiver is still widely-used [11, 12, 13, 14, 15]. One of its variant of FFT receiver is auto-correlation based receiver $[16,17,18,19,20]$. In recent years, the low probability of intercept (LPI) radars such as frequency modulated continuous wave (FMCW) radar [21, 22, 23] and its countermeasure have become a very active research area and an auto-correlation based receiver is effective at detecting the FMCW radar [23].

As a wideband receiver should detect concurrent signals,

${ }^{1}$ Defense Engineering Corporation, Beavercreek, Ohio, USA

2 Department of Electrical and Computer Engineering, Miami University, Oxford, Ohio, USA

a) chengc@miamioh.edu

DOI: $10.1587 /$ elex.18.20200453

Received December 28, 2020

Accepted January 14, 2021

Publicized January 22, 2021

Copyedited February 25, 2021

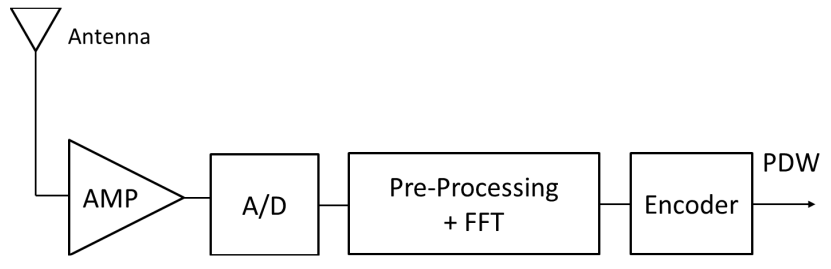

Fig. 1 Wideband receiver processing diagram.

it is a common practice to multiply signals with a window function to reduce signal's spectral leakage thus enabling the receiver to detect signals with large power difference. For an auto-correlation based receiver, the windowing can be performed before the auto-correlation or after the autocorrelation. The former approach is referred to as modified periodogram and the latter is referred to the Blackman-Tucky method. Although both of them are popular signal spectrum estimation techniques, to the best authors' knowledge, no one has compared their performance in the applications of wideband receiver. In this article, a thorough comparison is reported and it is concluded that modified periodogram can better improve the receiver's capability of detecting simultaneous signals with large power difference at the expense of reduced Probability of Detection (POD) for weak signals and capability of separating signals with close frequencies. The results presented in this article can provide engineers in the field of wideband receiver a useful guideline.

The rest of this article is organized as followings. Section 2 describes the concepts of modified periodogram and Blackman-Tucky method and Section 3 introduces some key specifications of wideband receiver. Simulation setting is described in Section 4 and simulation results are presented in Section 5. Section 6 concludes this article.

\section{Method}

Based on Wiener-Khinchin theorem, a wide-sense stationary random signal's power spectrum can be calculated via taking Fourier transforms of its auto-correlation [24]. The auto-correlation of a signal, $x[n]$, is defined as

$$
r_{x}[k]=\lim _{N \rightarrow \infty} \frac{1}{2 N+1} \sum_{n=-N}^{N} x[n+k] x^{*}[n] .
$$

In practice, only a finite segment of signal can be used in the analysis; the auto-correlation of an $N$-point sequence, $x[n]$, is calculated as:

$$
\tilde{r}_{x}[k]=\frac{1}{N} \sum_{n=0}^{N-1} x[n+k] x^{*}[n] .
$$




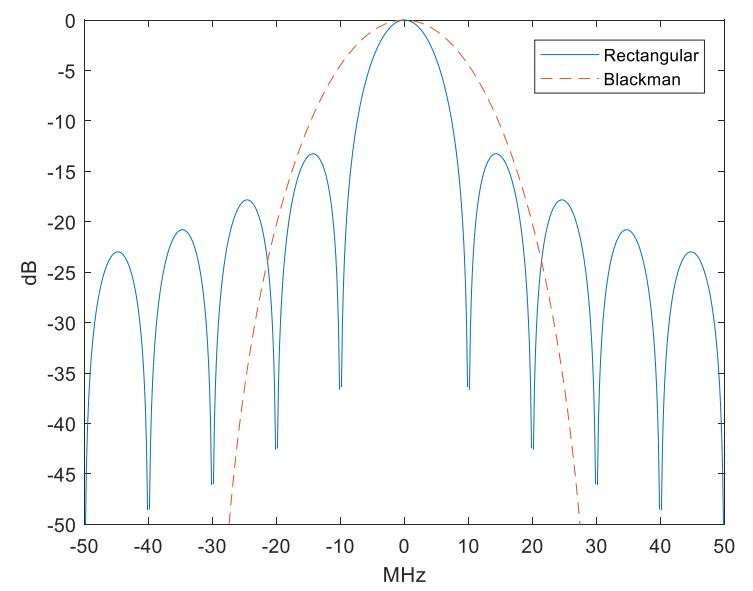

Fig. 2 Spectrum of a rectangular window and Blackman window. Blackman window has a wider mainlobe but lower sidelobes.

Since the length of $x[n]$ is finite, $\tilde{r}_{x}[k]$ equals zero outside the range of $[-N+1, N-1]$. The signal's periodogram, or power spectrum estimate, is calculated as,

$$
P_{x}\left(e^{j \omega}\right)=\sum_{k=-N+1}^{N-1} \tilde{r}_{x}[k] e^{-j k \omega} .
$$

It can be shown that

$$
P_{x}\left(e^{j \omega}\right)=\frac{1}{N}\left|X_{N}\left(e^{j \omega}\right)\right|^{2}
$$

where $X_{N}\left(e^{j \omega}\right)$ is the discrete-time Fourier transform of the $N$-point sequence $x[n]$.

The periodogram is an estimate of a random signal's power spectrum using a finite amount of samples and its expected value is the convolution of the true signal power spectrum and the square of a rectangular window's Fourier transform. The spectrum of a $0.1 \mu$ s rectangular window is shown in Fig. 2. Since the spectrum of a rectangular window has high sidelobes, a weak signal may not be detectable in the presence of a strong signal. To solve this issue, a window with low sidelobes is applied to the data before the auto-correlation [24].

A Blackman window [25, 26], whose spectrum is also shown in Fig. 2, can be a good choice and is used in this study. The periodogram obtained with data windowed by a non-rectangular window is referred to as the modified periodogram.

Although the expected value of the periodogram approaches the true signal power spectrum as the length of data increases, its variance does not. In other words, the periodogram is not a consistent estimate. One way to solve this issue is to take a long sequence of data, cut it into several segments, calculate the periodogram of each segment, and then average the periodograms. However, it might not always be feasible to acquire a long sequence of data.

Another way to reduce periodogram's variance is multiplying the auto-correlation by a window function before calculating its discrete-time Fourier transform. The logic behind this operation is to reduce the weight of auto-correlation results with higher variance in Eq. (2). Higher variance corresponds to where $\mathrm{k}$ is close to $\pm(\mathrm{N}-1)$ due to a smaller number of terms to be added. This method is referred to as the Blackman-Tucky method. In this study, the Blackman window is used to implement Blackman-Tucky method.

\section{Wideband receiver key specifications}

\subsection{Sensitivity}

The sensitivity of a wideband receiver is defined differently from the sensitivity of a communications receiver. In communications, the receiver's sensitivity is often defined as the minimum Signal to Noise Ratio (SNR) requirement to maintain a predefined Bit Error Rate (BER) [27]. For a wideband receiver, the definition of its sensitivity involves a False Alarm Rate (FAR) and a POD.

The FAR is the probability of detecting non-existent signals and the POD is the probability of detecting a real signal. It is desired to have a low FAR and a high POD. However, these two goals contradict with each other. Therefore, it is common practice to define a maximum FAR first and then to maximize the POD under the constraint that the FAR must be lower than the predefined upper limit.

In an FFT-based wideband receiver, a threshold is usually pre-determined based on a desired FAR [2]. Only signals whose FFT magnitude is above the threshold are considered detected signals. The easiest way to increase the wideband receiver's POD is to lower its threshold. However, a lower threshold will increase the FAR which is undesirable since a false alarm will trigger actions such as jamming a non-existent signal emitter thus wasting resources. In this study, the receiver's sensitivity is defined as the receiver's minimum SNR requirement to achieve a 90\% POD while maintaining a $10^{-7}$ FAR.

\subsection{Instantaneous dynamic range $[28,29,30]$}

For an FFT-based receiver, when a strong signal whose frequency is not a multiple of the FFT frequency resolution, or the strong signal does not occupy the full FFT window, the spectral leakage of the strong signal will increase the noise floor and may prevent the detection of a weak signal. The maximum power difference between two simultaneous signals whose PODs are larger than $90 \%$ is referred to as Instantaneous Dynamic Range (IDR) [2]. It is noteworthy that the IDR depends on the frequency separation of two signals as demonstrated later in this paper.

An ideal EW receiver should have a high sensitivity and a high IDR and these two specifications will be used to evaluate the signal processing algorithms considered in this study.

\section{Simulation settings}

A simulation is conducted to evaluate the performance difference between applying a window before or after autocorrelation as described in Section 2. In the simulation the test signal is a sinusoidal signal sampled at $2.56 \mathrm{GHz}$ with a frequency between $80 \mathrm{MHz}$ and $1.18 \mathrm{GHz}$, and each signal segment is 256 points long. Three methods are evaluated in the simulation.

- Auto-correlation is calculated and then the FFT is conducted upon the auto-correlation result. It serves as the baseline and is referred to as $A C$ in figures.

- The signal is multiplied with a Blackman window first 
before the auto-correlation is taken and the FFT is conducted upon the auto-correlation result. This is the modified periodogram method and is referred to as Window-AC in figures.

- The auto-correlation is calculated first and a Blackman window applied to the auto-correlation result. Afterwards, the FFT is conducted upon the product. This is the Blackman-Tucky method and referred to as $A C$ Window in figures.

A Gaussian noise signal with unit variance is first used as the input to determine the detection threshold to guarantee a $10^{-7}$ FAR.

\section{Results and discussion}

To determine receiver's sensitivity, the signal frequency is randomly chosen and 10,000 tests were run at each SNR setting to determine the receiver's POD vs. SNR. A signal is detected if its FFT magnitude is above the threshold and difference between the estimated frequency and real frequency is less than $10 \mathrm{MHz}$. The simulation result is illustrated in Fig. 3.

For the IDR study, two signals are used. One strong signal with an SNR set at $45 \mathrm{~dB}$ and its frequency is randomly selected. The second signal's power is set below the strong signal's power. The frequency separation is set as a controlled variable so we can see the effect of frequency separation on IDR. For each power difference and frequency offset setting, 5,000 simulations were run to determine the SNR requirement for the second signal to be detected with $90 \%$ of probability. The difference between strong signal's SNR and the SNR requirement for the second signal is the receiver's IDR at a specific frequency separation.

Like the sensitivity test, the signal is detected if its FFT magnitude is above the threshold and the frequency estimation error is less than $10 \mathrm{MHz}$. The IDR simulation results are provided in Fig. 4.

Fig. 3 and Fig. 4 show that the modified periodogram (Window-AC) improves the system IDR significantly when the frequency difference between two signals is more than $35 \mathrm{MHz}$. The Blackman-Tucky method (AC-Window) only improves IDR moderately. However, when the signal frequency difference is small the receiver using the modified periodogram method cannot detect the second signal.

The receiver using modified periodogram is also less sensitive. Compared to the other two receivers, the modified periodogram method needs a higher SNR (about 3dB) to reach $90 \%$ POD.

The reason behind the performance difference can be explained with Fig. 2. The Blackman window has a much wider mainlobe than the rectangular window. As the result, the convolution of the Blackman window spectrum with signal spectrum will spread signal power over several FFT bins and signals whose frequencies are close combine as one signal. This point is clearer through observing the output FFT magnitude spectra of the three receivers shown in Fig. 5. For the same signal, the maximum FFT magnitude of the receiver using modified periodogram is much lower than the other two receivers' output.

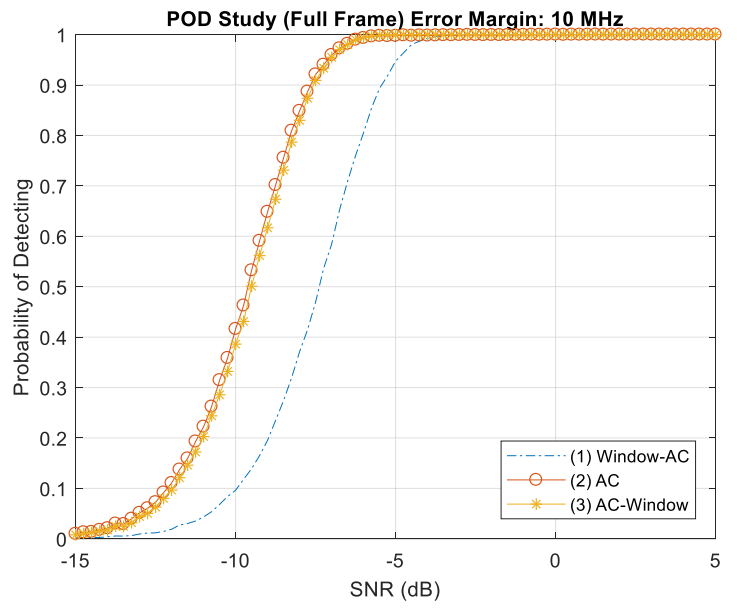

Fig. 3 Probability of detection vs. SNR. Sensitivity is reduced for Window-AC method.

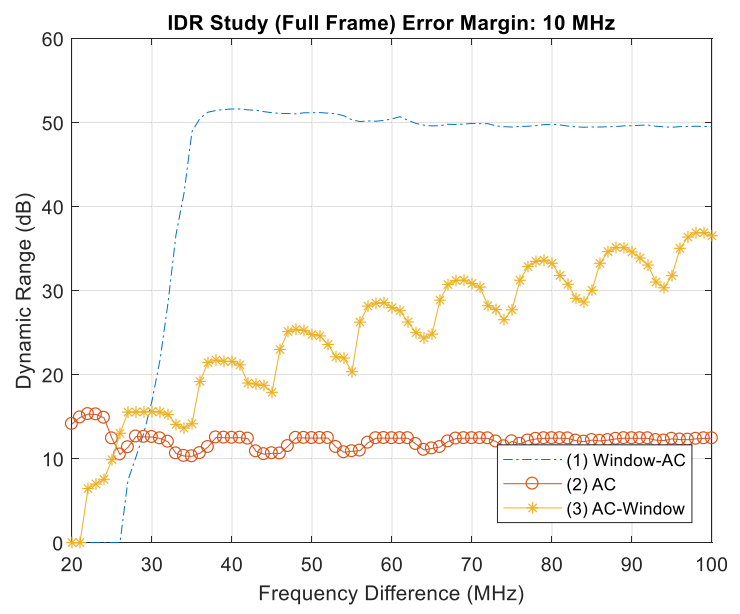

Fig. 4 Instantaneous dynamic range vs. SNR. Instantaneous dynamic range is better for $\mathrm{AC}$ and $\mathrm{AC}-W$ indow at lower frequencies.

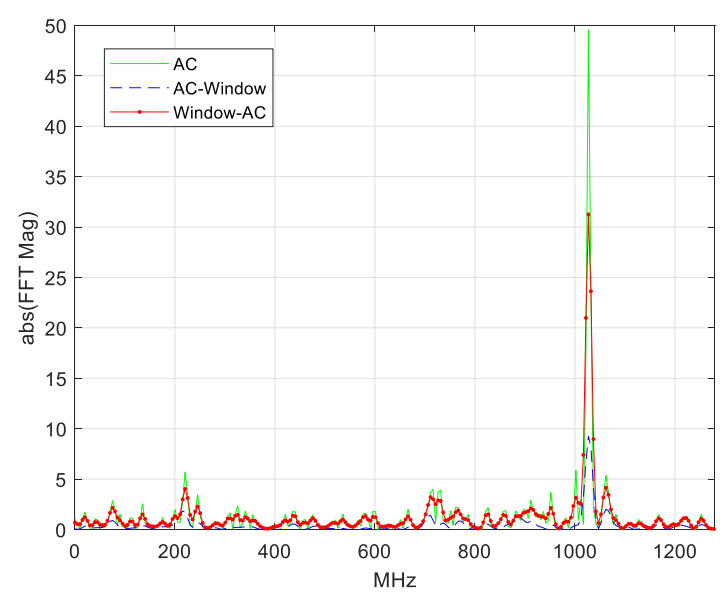

Fig. 5 Magnitude spectra of the three receivers under test. You may include display items in a final PDF version of your manuscript but do not exceed 4 MB file size.

Therefore, based on the simulation results, the modified periodogram can significantly improve a receiver's IDR at the expense of receiver's ability to distinguish signals with close frequencies and a receiver's sensitivity (about $3 \mathrm{~dB}$ in this specific case). The Blackman-Tucky method does not improve system IDR by a large margin but it does not 
sacrifice receiver's sensitivity significantly and it does not reduce receiver's capability of separating signals with close frequencies as much as the modified periodogram does.

It should be mentioned that different windows might be used for the wideband receiver but the conclusion about the performance difference between modified periodogram and Blackman-Tucky method made in this article is independent of window type.

\section{Conclusion}

Although applying a window has been used to improve the wideband receiver's IDR and both modified - periodogram and Blackman - Tucky methods have been used to estimate a random signal's spectrum this article, to the authors' knowledge, is the first to compare the performance difference for wideband receiver applications. The finding of this research is that the modified periodogram can better improve a receiver's capability of detecting signals with a significant power difference at the expense of reduced receiver sensitivity and frequency resolution for signal separation.

\section{References}

[1] J. Tsui: Digital Techniques for Wideband Receivers (SciTech Publishing, Raleigh, 2004) 2nd ed.

[2] J. Tsui: Special Design Topics in Digital Wideband Receivers (Artech House, Boston, 2010).

[3] A. Spezio: "Electronic warfare systems," IEEE Trans. Microw. Theory Techn. 50 (2002) 633 (DOI: 10.1109/22.989948).

[4] G. López-Risueňo, et al:: "Digital channelized receiver based on time-frequency analysis for signal interception," IEEE Trans. Aerosp. Electron. Syst. 41 (2005) 879 (DOI: 10.1109/TAES.2005.1541437).

[5] D. Adamy: EW 101: A First Course in Electronic Warfare (Artech House, Boston, 2001).

[6] R. Poisel: Target Acquisition in Communication Electronic Warfare Systems (Artech House, Boston, 2003).

[7] D.R. Zahirniak, et al.: "A hardware-efficient, multirate, digital channelized receiver architecture," IEEE Trans. Aerosp. Electron. Syst. 34 (1998) 137 (DOI: 10.1109/7.640270).

[8] S.-Y. Xie, et al.: "FPGA-based ultra-fast and wideband instantaneous frequency measurement receiver," IEICE Electron. Express 11 (2014) 1 (DOI: 10.1587/elex.11.20140263).

[9] C.-H. Cheng: "Wideband receiver design using frequency-dependent magnitude/phase mismatch," IEICE Electron. Express 11 (2014) 1 (DOI: 10.1587/elex.11.20140238)

[10] J. Kim, et al.: "The evolution of channelization receiver architecture: principles and design challenges," IEEE Access 5 (2017) 25385 (DOI: 10.1109/ACCESS.2017.2772810)

[11] M. Lee, et al.: "Analog auto-correlation based receiver architecture for radar systems," Proc. Military Communications Conference (2010) (DOI: 10.1109/MILCOM.2010.5680196).

[12] L. Po, et al.: "Design and implementation of a wideband digital reconnaissance receiver," 2009 IET International Radar Conference (2009) 1 (DOI: 10.1049/cp.2009.0302).

[13] B. Albaker and N. Rahim: "Signal acquisition and parameter estimation of radio frequency pulse radar using novel method," IETE Journal of Research 55 (2009) 128.

[14] M.A. Sanchez, et al.: "Implementing FFT-based digital channelized receivers on FPGA platforms," IEEE Trans. Aerosp. Electron. Syst. 44 (2008) 1567 (DOI: 10.1109/TAES.2008.4667732).

[15] P.W. East: "Fifty years of instantaneous frequency measurement," IET Radar, Sonar, and Navigation 6 (2012) 112 (DOI: 10.1049/ iet-rsn.2011.0177).

[16] S. Li, et al.: "Accurate measurement of radar carrier frequency," Proc. Int. Conf. Microwave and Millimeter Wave Technology (2007) 1 (DOI: 10.1109/ICMMT.2007.381513).
[17] B. Barshan and B. Eravci: "Automatic radar antenna scan type recogntion in electronic warfare," IEEE Trans. Aerosp. Electron. Syst. 48 (2012) 2908 (DOI: 10.1109/TAES.2012.6324669).

[18] J. Helton, et al.: "FPGA-based $1.2 \mathrm{GHz}$ bandwidth digital instantaneous frequency measurement receiver," Proc. Int. Symposium on Quality Electronic Design (2008) 568 (DOI: 10.1109/ISQED.2008. 4479798).

[19] B.D. Rigling and C. Roush: "ACF-based classification of phase modulated waveforms," Proc. Int. Radar Conference (2010) 287 (DOI: 10.1109/RADAR.2010.5494610).

[20] M. Pausini and G.J.M. Janssen: "Analysis and comparison of autocorrelation receivers for IR-UWB signals based on differential detection," Proc. IEEE International Conference on Acoustics, Speech, and Signal Processing (2004) 513 (DOI: 10.1109/ICASSP.2004.1326876).

[21] Y.-S. Won, et al.: "Method to improve degraded range resolution due to non-ideal factors in FMCW radar," IEICE Electron. Express 16 (2019) 1 (DOI: 10.1587/elex.15.20180924).

[22] T. Miura, et al.: "FMCW linear cell radar interference mitigation through control of signal delay in radio-over-fiber links," IEICE Electron. Express 17 (2020) 1 (DOI: 10.1587/elex.17.20200228).

[23] H. Chen, et al.: "Digital signal processing for a level measurement system based on FMCW radar,' IEEE International Conference on Control and Automation (2007) 2843 (DOI: 10.1109/ICCA.2007. 4376881).

[24] M.H. Hayes: Statistical Digital Signal Processing and Modeling (Wiley, New York, 1996).

[25] F.J. Harris: "On the use of windows for harmonic analysis with the discrete Fourier transform," Proc. IEEE 66 (1978) 51 (DOI: 10.1109/PROC.1978.10837).

[26] S.K. Mitra: Digital Signal Processing A Computer-Based Approach (McGraw-Hill, New York, 2006) 3rd ed.

[27] B. Sklar: Digital Communications: Fundamentals and Applications (Prentice Hall, Upper Saddle River, 2001).

[28] J. Grajal, et al.: "Analysis and chacterization of a monobit receiver for electronic warfare,” IEEE Trans. Aerosp. Electron. Syst. 39 (2003) 244 (DOI: 10.1109/TAES.2003.1188907).

[29] C. Pandolfi, et al.: "Comparison of analog IFM and digital frequency measurement receivers for electronic warfare," Proc. 7th European Radar Conference (2010) 232.

[30] S.R. Benson and C.-I.H. Chen: "Adaptive thresholding for high dualtone signal instantaneous dynamic range in digital wideband receiver," 2010 IEEE Instrumentation \& Measurement Technology Conference Proceedings (2010) 616 (DOI: 10.1109/IMTC.2010.5488140). 